\title{
Upper Gingival Verrucous Carcinoma
}

National Cancer Institute

\section{Source}

National Cancer Institute. Upper Gingival Verrucous Carcinoma. NCI Thesaurus. Code C5917.

A verrucous carcinoma of the oral cavity that arises from the upper gingiva. 